Supporting information for

\title{
Defect Characterization Using Raw Admittance Spectroscopy
}

\author{
Jian V. Li* \\ Department of Aeronautics and Astronautics, National Cheng Kung University \\ 1 University Road, Tainan, 70101 Taiwan \\ Corresponding author: \\ Email: jianvli@ncku.edu.tw
}




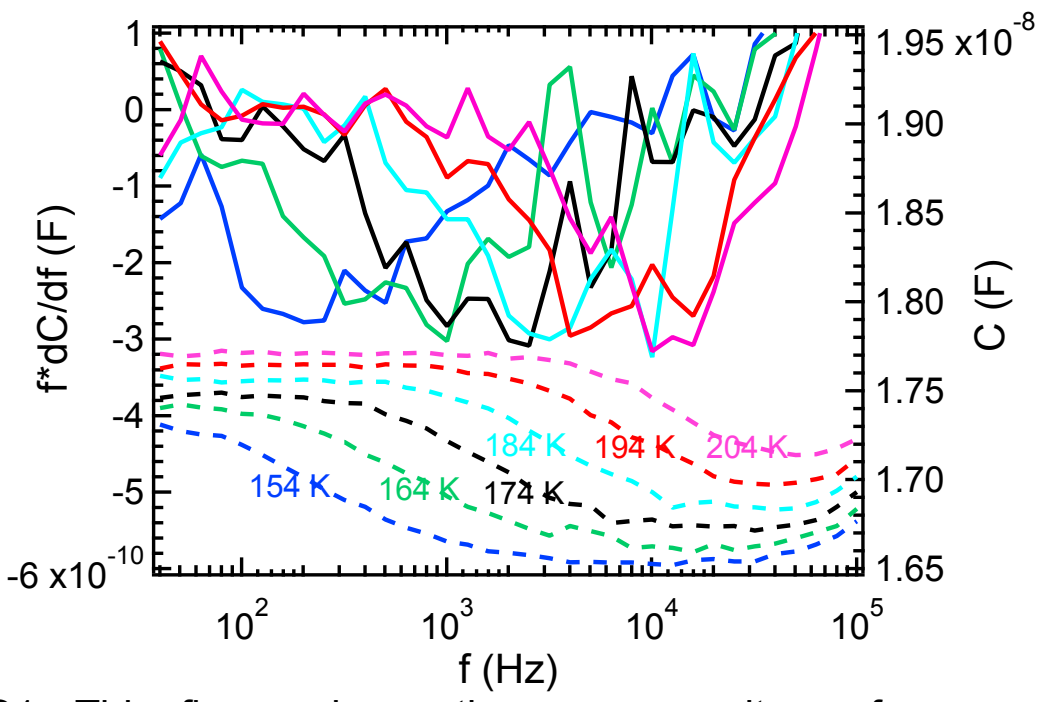

Figure $\mathrm{S} 1$. This figure shows the raw capacitance-frequency spectra (dashed lines) measured from a GaAs solar cell device grown by hydride vapor phase epitaxy at several temperatures and the corresponding frequency derivative $f^{*} \mathrm{dC} / \mathrm{df}$ spectra thereof (solid lines, using the same coloring scheme as the C-f spectra). Due to the numerical differentiation, the $f^{*} \mathrm{dC} / \mathrm{df}$ spectra exhibit quite poor signal-to-noise ratios and can barely reveal a defect signature (negative peak). Extracting peak positions in these spectra is a difficult task. Consequently, the conventional APL method of plotting the Arrhenius plot and extracting $E_{a}$ and $v_{0}$ is unreliable if not impossible. 


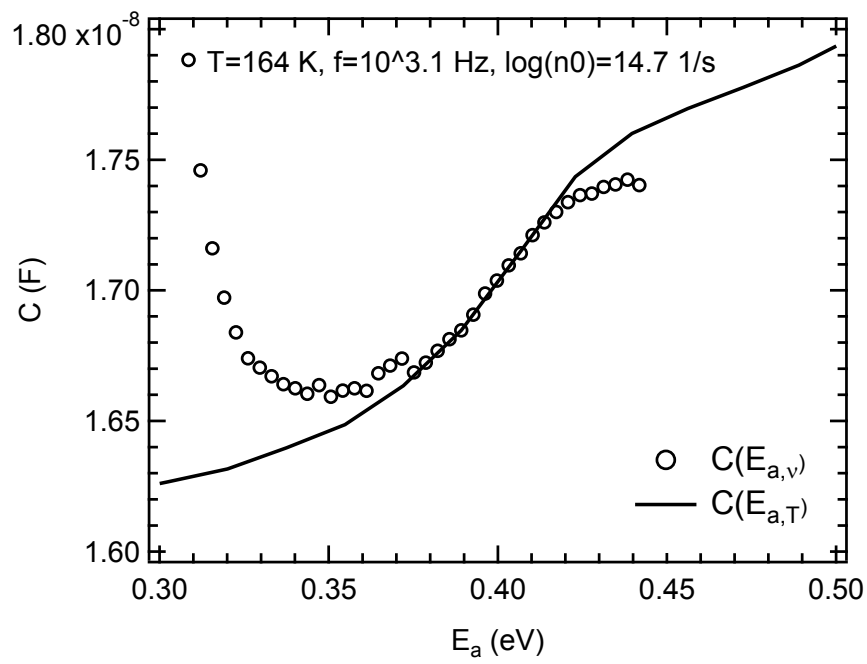

Figure S2. Figure S2 shows the application of the activation energy projection variation of the ATM method to transform and match the isothermal (symbols) and iso-rate (line) scans using data shown in Figure S1. The new ATM method described in this work directly uses the capacitance (i.e., the raw admittance data), which do not suffer the degradation of signalto-noise ratio due to the numerical differentiation. The ATM method is able to achieve a good agreement between $C\left(E_{a, v}\right)$ and $C\left(E_{a, T}\right)$ for a reliable extraction of $\log _{10}\left(v_{0}\right)=14.71 / \mathrm{s}$ and $E_{a}=0.378 \mathrm{eV}$ per $E q(1)$ at the fixed temperature and rate of $164 \mathrm{~K}$ and $1259 \mathrm{~Hz}$, respectively. 


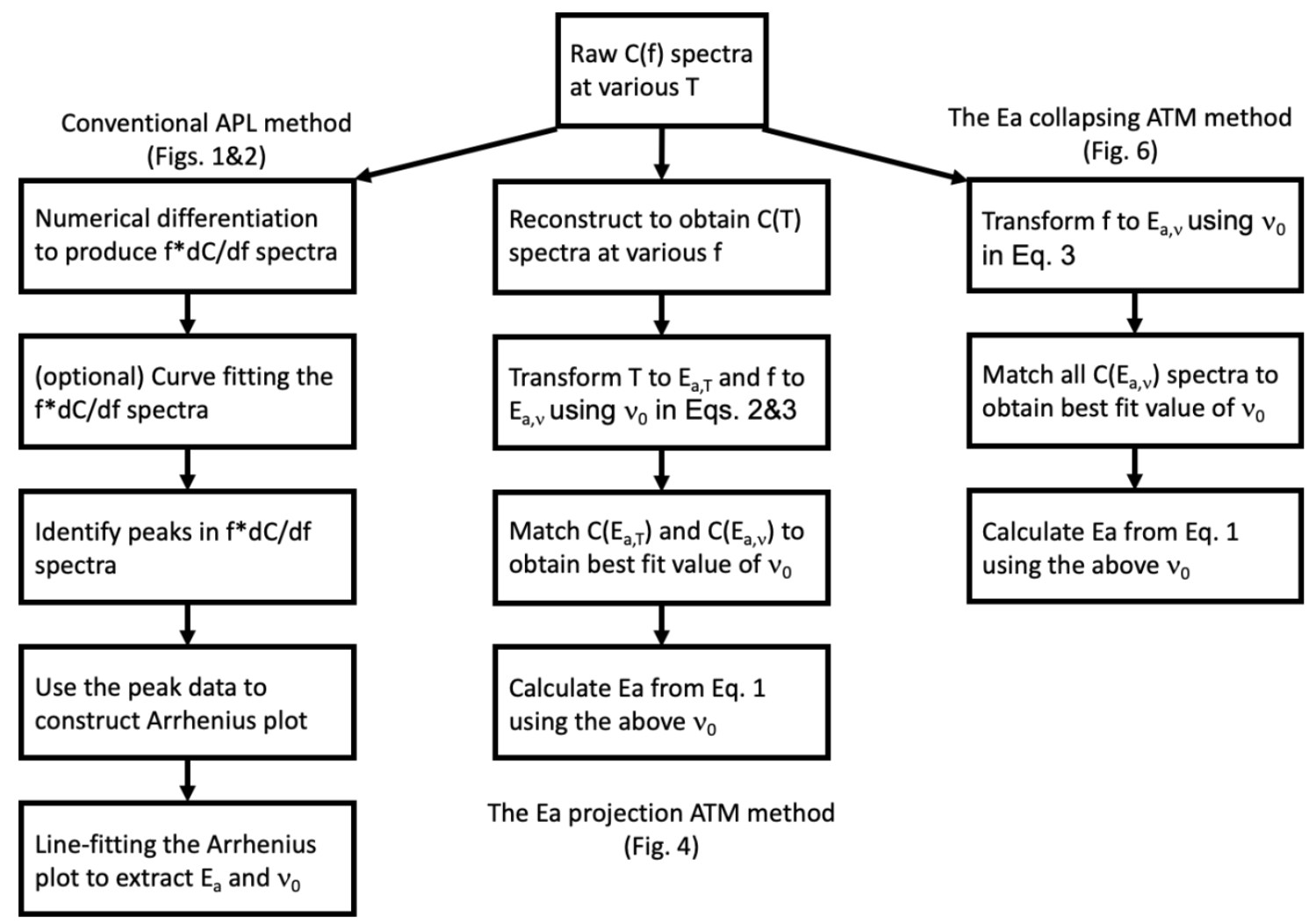

Figure S3. A diagram comparing the processing steps of the APL method (left column) and two variations (middle and right columns) of the ATM method. The ATM method takes a radically different approach to extract $E_{a}$ and $v_{0}$, possibly with fewer steps. Because of the reduced processing complexity (no numerical differentiation) and steps, the ATM method may save computing and analysis time compared to the conventional APL method 